\title{
Extensivity, entropy current, area law, and Unruh effect
}

\author{
F. Becattini and D. Rindori \\ Università di Firenze and INFN Sezione di Firenze, \\ Via G. Sansone 1, Sesto Fiorentino, I-50019 Florence, Italy
}

(Received 1 April 2019; published 21 June 2019)

\begin{abstract}
We present a general method to determine the entropy current of relativistic matter at local thermodynamic equilibrium in quantum statistical mechanics. Provided that the local equilibrium operator is bounded from below and its lowest lying eigenvector is non-degenerate, it is proved that, in general, the logarithm of the partition function is extensive, meaning that it can be expressed as the integral over a threedimensional space-like hypersurface of a vector current, and that an entropy current exists. We work out a specific calculation for a nontrivial case of global thermodynamic equilibrium, namely, a system with constant comoving acceleration, whose limiting temperature is the Unruh temperature. We show that the integral of the entropy current in the right Rindler wedge is the entanglement entropy.
\end{abstract}

DOI: 10.1103/PhysRevD.99.125011

\section{INTRODUCTION}

In recent years there has been a considerable interest in the foundations of relativistic hydrodynamics. One of the key quantities is the so-called entropy current $s^{\mu}$, which is one of the postulated ingredients of Israel's formulation [1]. Therein, the entropy current plays a very important role because, as its divergence ought to be positive, its form entails the constitutive equations of the conserved currents (stress-energy tensor, charged currents) as a function of the gradients of the intensive thermodynamic parameters. Over the last decade, there have been a very large number of studies where the structure of the entropy current in relativistic hydrodynamics was involved; see, for example, Refs. [2-9]. On the other hand, there have been attempts [10] to formulate relativistic hydrodynamics without an entropy current.

In most of these studies, the structure of the entropy current is postulated based on some classical form of the thermodynamics laws supplemented by more elaborate methods to include dissipative corrections [11,12], but, strictly speaking, it is not derived. The ultimate reason for the apparent insufficient definition is that the entropy current (unlike the stress-energy tensor or charged currents) in the familiar quantum field theory is not the mean value of a local operator built with quantum fields (in the generating functional approach, this difference can be rephrased by saying that the entropy current cannot be

Published by the American Physical Society under the terms of the Creative Commons Attribution 4.0 International license. Further distribution of this work must maintain attribution to the author(s) and the published article's title, journal citation, and DOI. Funded by SCOAP ${ }^{3}$. obtained by taking functional derivatives with respect to some external source). ${ }^{1}$ Another reason for this indeterminacy is the fact that, while in quantum statistical mechanics the total entropy has a precise definition in terms of the density operator (von Neumann formula) $S=-\operatorname{tr}(\hat{\rho} \log \hat{\rho})$, the entropy current, which should be a more fundamental quantity than the total entropy in a general-relativistic framework, does not.

In this work, we will show that it is indeed possible to provide a rigorous definition of the entropy current in quantum-relativistic statistical mechanics and thereby to derive its form in a situation of local thermodynamic equilibrium, which is the underlying assumption of relativistic hydrodynamics. The method is based on the definition of a density operator at local thermodynamic equilibrium put forward in the late 1970s by Zubarev and van Weert [14,15] and reworked more recently in Refs. [16,17]. The key step in showing that an entropy current exists is the proof that, in a relativistic theory, the logarithm of the partition function is extensive, i.e., it can be expressed as an integral over a threedimensional (3D) space-like hypersurface of a four-vector field. We will provide, to the best of our knowledge, the first general proof of this usually tacitly understood hypothesis under very general conditions.

After the general method, we will present a specific, nontrivial calculation of the entropy current which is especially interesting as it is related to the Unruh effect. Indeed, we will show that in the Minkowski vacuum an acceleration involves a nonvanishing finite entropy current

\footnotetext{
${ }^{1}$ Indeed, in Ref. [8] the authors proposed a method to obtain an entropy current from a supersymmetric generating functional, which was also the idea of Ref. [13].
} 
in some region which, once integrated, gives rise to its total entanglement entropy.

\section{A. Notation}

In this paper we adopt the natural units, with $\hbar=$ $c=K=1$. The Minkowskian metric tensor is $g=$ $\operatorname{diag}(1,-1,-1,-1)$ and for the Levi-Civita symbol we use the convention $\varepsilon^{0123}=1$. Operators in Hilbert space will be denoted by an upper hat, e.g., $\hat{\mathrm{R}}$.

We will use the relativistic notation with repeated indices assumed to be saturated, and contractions of indices will sometimes be denoted with dots, e.g., $b \cdot \hat{P}=b_{\mu} \hat{P}^{\mu}$ or $\varpi: \hat{J}=\varpi_{\mu \nu} \hat{J}^{\mu \nu}$.

$\nabla_{\mu}$ denotes the covariant derivative in curved space-time.

\section{EXTENSIVITY AND ENTROPY CURRENT}

In quantum statistical mechanics, the state of a physical system is described by a density operator $\hat{\rho}$. At global thermodynamic equilibrium, $\hat{\rho}$ is determined, according to the maximum entropy principle, by maximizing the entropy $S=-\operatorname{tr}(\hat{\rho} \log \hat{\rho})$ with a set of constraints, such as fixing the value of the energy and some possible charges. Similarly, in a situation of local thermodynamic equilibrium (LTE), the density operator $\hat{\rho}_{\mathrm{LE}}$ is determined by maximizing the entropy constrained with energy-momentum and charge densities. In a relativistic framework, the LTE density operator definition depends on a 3D space-like hypersurface $\Sigma$ where the densities are given; the fully covariant expression reads [14]

$$
\hat{\rho}_{\mathrm{LE}}=\frac{1}{Z_{\mathrm{LE}}} \exp \left[-\int_{\Sigma} \mathrm{d} \Sigma_{\mu}\left(\hat{T}^{\mu \nu} \beta_{\nu}-\zeta \hat{j}^{\mu}\right)\right],
$$

where

$$
Z_{\mathrm{LE}}=\operatorname{tr}\left(\exp \left[-\int_{\Sigma} \mathrm{d} \Sigma_{\mu}\left(\hat{T}^{\mu \nu} \beta_{\nu}-\zeta \hat{j}^{\mu}\right)\right]\right)
$$

is the partition function. In Eq. (1), $\hat{T}^{\mu \nu}$ is the stress-energy tensor, $\hat{j}^{\mu}$ is a conserved current, ${ }^{2} \beta_{\nu}$ is the time-like fourtemperature vector, whose magnitude is the inverse comoving temperature

$$
T=\frac{1}{\sqrt{\beta^{2}}}
$$

while its direction defines a flow velocity [16]

$$
u^{\mu}=\frac{\beta^{\mu}}{\sqrt{\beta^{2}}},
$$

\footnotetext{
${ }^{2}$ We do not consider anomalous currents in this work. However, we reckon that the proposed method can be extended to anomalous currents as well by using the approach of Ref. [18].
}

and $\zeta$ is a scalar that represents the ratio between the comoving chemical potential and comoving temperature, i.e.,

$$
\zeta=\frac{\mu}{T}
$$

With the density operator (1), one can calculate the total entropy as a function of the four-temperature, which turns out to be

$$
S=\log Z_{\mathrm{LE}}+\int_{\Sigma} \mathrm{d} \Sigma_{\mu}\left(\left\langle\hat{T}^{\mu \nu}\right\rangle_{\mathrm{LE}} \beta_{\nu}-\zeta\left\langle\hat{j}^{\mu}\right\rangle_{\mathrm{LE}}\right)
$$

where by \langle\rangle$_{\mathrm{LE}}$ we mean the expectation value calculated with $\hat{\rho}_{\mathrm{LE}}$. The expectation value of a local operator $\hat{O}(x)$ is then a local intensive quantity depending only on $x$ provided that $x$ belongs to the hypersurface $\Sigma$ in the definition of the operator (1). In particular, if $\Sigma$ is a hyperplane $t=$ const, this means that the time component $x^{0}$ of $x$ must be equal to $t$. This condition will be henceforth understood. For an entropy current to exist, meaning that

$$
S=\int_{\Sigma} \mathrm{d} \Sigma_{\mu} s^{\mu}
$$

it can be readily seen from Eq. (4) that $\log Z_{\mathrm{LE}}$ must be extensive, namely, that there must be a four-vector $\phi$, called the thermodynamic potential current, such that

$$
\log Z_{\mathrm{LE}}=\int_{\Sigma} \mathrm{d} \Sigma_{\mu} \phi^{\mu}
$$

so that, comparing Eqs. (4) and (5),

$$
s^{\mu}=\phi^{\mu}+\left\langle\hat{T}^{\mu \nu}\right\rangle_{\mathrm{LE}} \beta_{\nu}-\zeta\left\langle\hat{j}^{\mu}\right\rangle_{\mathrm{LE}} .
$$

Notice that both $\phi$ and $s$ are defined up to an arbitrary fourvector tangent to the space-like hypersurface $\Sigma$.

The existence of the thermodynamic potential current and (as a consequence) the entropy current is usually assumed. One of the goals of this work is to show that its existence can be proved under very general hypotheses. To begin with, let us modify the LTE density operator by introducing a dimensionless parameter $\lambda$,

$$
\hat{\rho}_{\mathrm{LE}}(\lambda)=\frac{1}{Z_{\mathrm{LE}}(\lambda)} \exp \left[-\lambda \int_{\Sigma} \mathrm{d} \Sigma_{\mu}\left(\hat{T}^{\mu \nu} \beta_{\nu}-\zeta \hat{j}^{\mu}\right)\right],
$$

so that for $\lambda=1$ we recover the actual LTE density operator. Since

$$
Z_{\mathrm{LE}}(\lambda)=\operatorname{tr}\left(\exp \left[-\lambda \int_{\Sigma} \mathrm{d} \Sigma_{\mu}\left(\hat{T}^{\mu \nu} \beta_{\nu}-\zeta \hat{j}^{\mu}\right)\right]\right),
$$

by taking the derivative of the trace we obtain 


$$
\frac{\partial \log Z_{\mathrm{LE}}(\lambda)}{\partial \lambda}=-\int_{\Sigma} \mathrm{d} \Sigma_{\mu}\left(\left\langle\hat{T}^{\mu \nu}\right\rangle_{\mathrm{LE}}(\lambda) \beta_{\nu}-\zeta\left\langle\hat{j}^{\mu}\right\rangle_{\mathrm{LE}}(\lambda)\right),
$$

and, by integrating both sides,

$$
\begin{aligned}
& \log Z_{\mathrm{LE}}-\log Z_{\mathrm{LE}}\left(\lambda_{0}\right) \\
& =-\int_{\lambda_{0}}^{1} \mathrm{~d} \lambda \int_{\Sigma} \mathrm{d} \Sigma_{\mu}\left(\left\langle\hat{T}^{\mu \nu}\right\rangle_{\mathrm{LE}}(\lambda) \beta_{\nu}-\zeta\left\langle\hat{j}^{\mu}\right\rangle_{\mathrm{LE}}(\lambda)\right)
\end{aligned}
$$

for some $\lambda_{0}$, where $\log Z_{\mathrm{LE}}(1)$ is the actual $\log Z_{\mathrm{LE}}$. If we then exchange the integrations in Eq. (7), we get

$$
\begin{aligned}
& \log Z_{\mathrm{LE}}-\log Z_{\mathrm{LE}}\left(\lambda_{0}\right) \\
& =-\int_{\Sigma} \mathrm{d} \Sigma_{\mu} \int_{\lambda_{0}}^{1} \mathrm{~d} \lambda\left(\left\langle\hat{T}^{\mu \nu}\right\rangle_{\mathrm{LE}}(\lambda) \beta_{\nu}-\zeta\left\langle\hat{j}^{\mu}\right\rangle_{\mathrm{LE}}(\lambda)\right) .
\end{aligned}
$$

Thus, if there exists a particular $\lambda_{0}$ such that $\log Z_{\mathrm{LE}}\left(\lambda_{0}\right)=0$, it is proved that $\log Z_{\mathrm{LE}}$ is extensive and, at the same time, we have a method to determine the thermodynamic potential current:

$$
\begin{aligned}
\log Z_{\mathrm{LE}} & =\int_{\Sigma} \mathrm{d} \Sigma_{\mu} \phi^{\mu} \\
\phi^{\mu} & =-\int_{\lambda_{0}}^{1} \mathrm{~d} \lambda\left(\left\langle\hat{T}^{\mu \nu}\right\rangle_{\mathrm{LE}}(\lambda) \beta_{\nu}-\zeta\left\langle\hat{j}^{\mu}\right\rangle_{\mathrm{LE}}(\lambda)\right) .
\end{aligned}
$$

At a glance, one would say that $\lambda=+\infty$ can make $Z_{\mathrm{LE}}(\lambda)$ very small. More thoroughly, one should study the spectrum of the local equilibrium operator in the exponent of Eq. (1), defined as

$$
\hat{\Upsilon} \equiv \int_{\Sigma} \mathrm{d} \Sigma_{\mu}\left(\hat{T}^{\mu \nu} \beta_{\nu}-\zeta \hat{j}^{\mu}\right) .
$$

It is easily realized that this operator, if $\zeta=0$, is simply the Hamiltonian divided by the temperature in the special case where $\beta=1 / T(1, \mathbf{0})$. Suppose that the local equilibrium operator $\hat{\Upsilon}$ is bounded from below, i.e., there exists a minimum eigenvalue $\Upsilon_{0}$ with a corresponding eigenvector $|0\rangle$, which is supposedly nondegenerate. In this case, by ordering the eigenvalues $\Upsilon_{0}<\Upsilon_{1}<\Upsilon_{2} \ldots$, and if the lowest eigenvector is nondegenerate, the trace can be written as

$$
\begin{aligned}
Z_{\mathrm{LE}}(\lambda) & =\operatorname{tr}\left(\mathrm{e}^{-\lambda \hat{\Upsilon}}\right) \\
& =\mathrm{e}^{-\lambda \Upsilon_{0}}\left(1-\mathrm{e}^{-\lambda\left(\Upsilon_{1}-\Upsilon_{0}\right)}-\mathrm{e}^{-\lambda\left(\Upsilon_{2}-\Upsilon_{0}\right)}-\cdots\right),
\end{aligned}
$$

so, if $\Upsilon_{0}=0$ and we let $\lambda \rightarrow+\infty$, we obtain the sought solution, that is,

$$
\lim _{\lambda \rightarrow+\infty} Z_{\mathrm{LE}}(\lambda)=1 \Rightarrow \lim _{\lambda \rightarrow+\infty} \log Z_{\mathrm{LE}}(\lambda)=0 .
$$

One can now take advantage of the invariance of the density operator (1) by subtraction of a constant number in the exponent. What we mean is that $\hat{\rho}$ (as well as the entropy) is invariant if we replace $\hat{\Upsilon}$ in Eq. (8) with

$$
\begin{aligned}
\hat{\Upsilon} \mapsto \hat{\Upsilon}-\Upsilon_{0}= & \hat{\Upsilon}-\langle 0|\hat{\Upsilon}| 0\rangle \\
= & \int_{\Sigma} \mathrm{d} \Sigma_{\mu}\left[\left(\hat{T}^{\mu \nu}-\left\langle 0\left|\hat{T}^{\mu \nu}\right| 0\right\rangle\right) \beta_{\nu}\right. \\
& \left.-\zeta\left(\hat{j}^{\mu}-\left\langle 0\left|\hat{j}^{\mu}\right| 0\right\rangle\right)\right]
\end{aligned}
$$

and calculate the partition function accordingly, that is, $Z^{\prime}=\operatorname{tr}\left(\exp \left[-\hat{\Upsilon}+\Upsilon_{0}\right]\right)$. Hence, the new $\lambda$-dependent partition function reads

$$
\begin{aligned}
Z_{\mathrm{LE}}^{\prime}(\lambda)= & \operatorname{tr}\left(\operatorname { e x p } \left\{-\lambda \int_{\Sigma} \mathrm{d} \Sigma_{\mu}\left[\left(\hat{T}^{\mu \nu}-\left\langle 0\left|\hat{T}^{\mu \nu}\right| 0\right\rangle\right) \beta_{\nu}\right.\right.\right. \\
& \left.\left.\left.-\zeta\left(\hat{j}^{\mu}-\left\langle 0\left|\hat{j}^{\mu}\right| 0\right\rangle\right)\right]\right\}\right) .
\end{aligned}
$$

We note in passing that the subtraction of the expectation value of $\hat{T}^{\mu \nu}$ in the lowest-lying eigenvector of $\hat{\Upsilon}$ does not imply that the thereby obtained operator $\hat{T}^{\mu \nu}-\left\langle 0\left|\hat{T}^{\mu \nu}\right| 0\right\rangle$ is the physically renormalized one. In fact, in the accelerated thermodynamic equilibrium case, the physical operator is obtained by subtracting the expectation value in the Minkowski vacuum [19], whereas the lowest-lying eigenvector is the so-called Rindler vacuum, as we will see in Sec. IV.

The new partition function is such that $Z_{\mathrm{LE}}^{\prime}(\infty)=1$, and the thermodynamic potential current is thus given by

$$
\begin{aligned}
\phi^{\mu}= & \int_{1}^{+\infty} \mathrm{d} \lambda\left[\left(\left\langle\hat{T}^{\mu \nu}\right\rangle_{\mathrm{LE}}(\lambda)-\left\langle 0\left|\hat{T}^{\mu \nu}\right| 0\right\rangle\right) \beta_{\nu}\right. \\
& \left.-\zeta\left(\left\langle\hat{j}^{\mu}\right\rangle_{\mathrm{LE}}(\lambda)-\left\langle 0\left|\hat{j}^{\mu}\right| 0\right\rangle\right)\right] .
\end{aligned}
$$

Consequently, the entropy current will be

$s^{\mu}=\phi^{\mu}+\left(\left\langle\hat{T}^{\mu \nu}\right\rangle_{\mathrm{LE}}-\left\langle 0\left|\hat{T}^{\mu \nu}\right| 0\right\rangle\right) \beta_{\nu}-\zeta\left(\left\langle\hat{j}^{\mu}\right\rangle_{\mathrm{LE}}-\left\langle 0\left|\hat{j}^{\mu}\right| 0\right\rangle\right)$.

These formulas show that the thermodynamic potential current is obtained by effectively integrating the temperature dependence of the currents, as $\lambda$ multiplies $\beta$ and $\zeta$. Even in the presence of first-order phase transitions, with discontinuities in the mean values of the stress-energy tensor and charged current as a function of the temperature, the integral is still feasible and makes perfect sense.

We can finally draw an important conclusion regarding the existence of the entropy current.

Theorem. If the spectrum of the local equilibrium operator (8) is bounded from below, and if the eigenvector corresponding to its lowest eigenvalue is nondegenerate, the logarithm of the partition function is extensive. Thus, we can obtain a thermodynamic potential current by integrating 
the difference between the expectation values of the stressenergy tensor and conserved currents and their expectation values in the lowest-lying eigenvector of the local equilibrium operator.

So, to solve the problem, one just needs to determine the mean values of the stress-energy tensor and the currents. We will see how this can be accomplished in a nontrivial case in Sec. IV.

\section{THERMODYNAMIC EQUILIBRIUM WITH ACCELERATION IN MINKOWSKI SPACE-TIME}

The local thermodynamic equilibrium density operator (1) can be promoted to global thermodynamic equilibrium when it becomes time independent or, in covariant language, independent of the space-like hypersurface $\Sigma$. This has been studied in detail elsewhere [20]. Global thermodynamic equilibrium occurs when $\zeta$ is constant and the four-temperature $\beta$ is a Killing vector, that is,

$$
\nabla_{\mu} \beta_{\nu}+\nabla_{\nu} \beta_{\mu}=0
$$

It is readily seen from Eqs. (9) and (10) that, in this case, if the stress-energy tensor and the currents are conserved, so are the thermodynamic potential current and the entropy current,

$$
\nabla_{\mu} \phi^{\mu}=0, \quad \nabla_{\mu} s^{\mu}=0,
$$

in agreement with the general condition that at global thermodynamic equilibrium the entropy production rate vanishes.

In Minkowski space-time the general solution of the Killing equation (11) is

$$
\beta_{\mu}=b_{\mu}+\varpi_{\mu \nu} x^{\nu}
$$

where $b$ is a constant four-vector and $\varpi$ is a constant antisymmetric tensor called the thermal vorticity. The latter can be expressed as the exterior derivative of the fourtemperature, i.e., $\varpi_{\mu \nu}=-\frac{1}{2}\left(\partial_{\mu} \beta_{\nu}-\partial_{\nu} \beta_{\mu}\right)$.

By using Eq. (12), one can obtain the general form of the density operator (1) in Minkowski space-time, that is,

$$
\hat{\rho}=\frac{1}{Z} \exp \left[-b_{\mu} \hat{P}^{\mu}+\frac{1}{2} \varpi_{\mu \nu} \hat{J}^{\mu \nu}+\zeta \hat{Q}\right],
$$

where $\hat{P}$ is the four-momentum operator, $\hat{J}$ is the boostangular momentum operator, and $\hat{Q}$ is the conserved charge associated to the current $\hat{j}$. Among the various solutions, a noteworthy one is the pure acceleration one,

$$
b_{\mu}=\frac{1}{T_{0}}(1, \mathbf{0}), \quad \varpi_{\mu \nu}=\frac{a}{T_{0}}\left(g_{0 \nu} g_{3 \mu}-g_{3 \nu} g_{0 \mu}\right),
$$

with constant parameters $a$ and $T_{0}$. This case has been studied in detail in Ref. [19] and corresponds to a fluid with four-temperature field

$$
\beta^{\mu}=\frac{a}{T_{0}}\left(z^{\prime}, 0,0, t\right)
$$

where $z^{\prime} \equiv z+1 / a$, and a four-acceleration field

$$
A^{\mu}=\frac{1}{z^{\prime 2}-t^{2}}\left(t, 0,0, z^{\prime}\right)
$$

It is readily found that the $\beta$ field (15) is time-like for $\left|z^{\prime}\right|>t$, light-like for $\left|z^{\prime}\right|=t$, and space-like for $\left|z^{\prime}\right|<t$. Hence, the hypersurfaces $\left|z^{\prime}\right|=t$ are two Killing horizons for $\beta$ and they break the space-time into four different regions: the region $|t|<z^{\prime}$ is called the right Rindler wedge (RRW), the region $|t|<-z^{\prime}$ is called the left Rindler wedge (LRW), while the regions $|t|>\left|z^{\prime}\right|$ are not of interest here. The proper temperature (2) in the RRW is singular on the light-cone boundary:

$$
T=\frac{T_{0}}{a} \frac{1}{\sqrt{z^{\prime 2}-t^{2}}} .
$$

It is also very useful to decompose the thermal vorticity tensor $\varpi$ into two space-like vector fields $\alpha$ and $w$ [21] by projecting onto the velocity field (3). In the global equilibrium case, they turn out to be parallel to the fouracceleration and the kinematic vorticity, respectively, and both are orthogonal to $u$ or $\beta$. In the pure acceleration case, it turns out that the kinematic vorticity vanishes and we are left with

$$
\varpi_{\mu \nu}=\alpha_{\mu} u_{\nu}-\alpha_{\nu} u_{\mu},
$$

where $\alpha^{\mu}=A^{\mu} / T$ is the four-acceleration divided by the comoving temperature. By using Eqs. (16), (15), and (2) to calculate $T$, it turns out that

$$
\alpha^{2}=\frac{A^{2}}{T^{2}}=\beta^{2} A^{2}=-\frac{a^{2}}{T_{0}^{2}},
$$

that is, $\alpha^{2}$ is constant.

In the pure acceleration case (14) with $\zeta=0$, the density operator (13) becomes

$$
\hat{\rho}=\frac{1}{Z} \exp \left[-\frac{\hat{H}}{T_{0}}+\frac{a}{T_{0}} \hat{K}_{z}\right]
$$

where $\hat{K}_{z}=\hat{J}_{30}$ is the boost generator along the $z$ direction. The operator $\hat{H}-a \hat{K}_{z}$ is stationary and can be regarded as the generator of translations along the flow lines of Eq. (15) [22]. 
The peculiarity of the four-temperature field (15) is that it vanishes altogether on the two-dimensional (2D) surface $t=0, z^{\prime}=0$, which makes it possible to factorize the density operator into two commuting operators involving the quantum field degrees of freedom on either side of $z^{\prime}=0$, i.e.,

$$
\begin{gathered}
\hat{\rho}=\hat{\rho}_{R} \otimes \hat{\rho}_{L}, \quad\left[\hat{\rho}_{R}, \hat{\rho}_{L}\right]=0, \\
\hat{\rho}_{R}=\frac{1}{Z_{R}} \exp \left[-\frac{\hat{\Pi}_{R}}{T_{0}}\right], \quad \hat{\rho}_{L}=\frac{1}{Z_{L}} \exp \left[\frac{\hat{\Pi}_{L}}{T_{0}}\right],
\end{gathered}
$$

with

$$
\hat{\Pi}_{R, L} \equiv \pm T_{0} \int_{z^{\prime} \gtrless 0} \mathrm{~d} \Sigma_{\mu} \hat{T}^{\mu \nu} \beta_{\nu}
$$

being the generator of translations along the flow lines, playing the role of the Hamiltonian. The factorization implies that, if $\hat{O}(x)$ is a local operator with $x$ in the RRW, its expectation value is independent of the field operators in the LRW.

Quantum field equations of motion, such as the KleinGordon equation, are solved in the RRW and LRW separately by introducing proper hyperbolic coordinatesthe Rindler coordinates $(\tau, x, y, \xi)$-where the "transverse" coordinates $\mathbf{x}_{T} \equiv(x, y)$ are the same as the Minkowski coordinates, and $(\tau, \xi)$ are related to $\left(t, z^{\prime}\right)$ by

$$
\tau \equiv \frac{1}{2 a} \log \left(\frac{z^{\prime}+t}{z^{\prime}-t}\right), \quad \xi \equiv \frac{1}{2 a} \log \left[a^{2}\left(z^{\prime 2}-t^{2}\right)\right]
$$

in the RRW. Inverting and plugging them into the Klein-Gordon equation, one finds the positive-frequency modes [23]

$$
\begin{aligned}
u_{\omega, \mathbf{k}_{T}}\left(\tau, \xi, \mathbf{x}_{T}\right) \equiv & \sqrt{\frac{1}{4 \pi^{4} a} \sinh \left(\frac{\pi \omega}{a}\right)} \\
& \times \mathrm{K}_{i \frac{\omega}{a}}\left(\frac{m_{T} \mathrm{e}^{a \xi}}{a}\right) \mathrm{e}^{-i\left(\omega \tau-\mathbf{k}_{T} \cdot \mathbf{x}_{T}\right)},
\end{aligned}
$$

where $\omega$ is a positive real number, $\mathbf{k}_{T} \equiv\left(k_{x}, k_{y}\right)$ is the "transverse" momentum, the K's are the modified Bessel functions, and $m_{T}^{2} \equiv \omega^{2}-\mathbf{k}_{T}^{2}$. The real scalar field can thus be expanded as

$\hat{\psi}\left(\tau, \xi, \mathbf{x}_{T}\right)=\int_{0}^{+\infty} \mathrm{d} \omega \int_{\mathbb{R}^{2}} \mathrm{~d}^{2} k_{T}\left(u_{\omega, \mathbf{k}_{T}} \hat{a}_{\omega, \mathbf{k}_{T}}^{R}+u_{\omega, \mathbf{k}_{T}}^{*} \hat{a}_{\omega, \mathbf{k}_{T}}^{R^{\dagger}}\right)$,

where $\hat{a}^{R^{\dagger}}$ and $\hat{a}^{R}$ are the creation and annihilation operators, respectively, and they satisfy the usual commutation relations. A similar field expansion holds in the LRW, with the important difference that the role of the creation and annihilation operators is interchanged, as a consequence of the fact that the boost generator, which plays the role of a time-translation generator, is pastoriented therein. The "Hamiltonians" $\hat{\Pi}$ turn out to be [19]

$$
\hat{\Pi}_{R, L}=\int_{0}^{+\infty} \mathrm{d} \omega \int_{\mathbb{R}^{2}} \mathrm{~d}^{2} k_{T} \hat{a}_{\omega, \mathbf{k}_{T}}^{\dagger R} \hat{a}_{\omega, \mathbf{k}_{T}}^{R, L} .
$$

The vacuum in the RRW is obtained by requiring it to be annihilated by all the operators $\hat{a}^{R}$, and it is denoted as $\left|0_{R}\right\rangle$. Similarly, the vacuum $\left|0_{L}\right\rangle$ in the LRW is the state annihilated by all $\hat{a}^{L}$, and the overall vacuum state $|0\rangle_{R} \equiv$ $\left|0_{R}\right\rangle \otimes\left|0_{L}\right\rangle$ is the so-called Rindler vacuum. As first pointed out by Fulling in Ref. [24], the Rindler vacuum does not coincide with the Minkowski vacuum. In fact, the Minkowski vacuum is a thermal state of free bosons with temperature $a / 2 \pi$-the well-known Unruh effect [25].

The thermal expectation values of relevant physical quantities in the RRW, for a free field, with the density operator (20) can be obtained once the thermal expectation values of products of creation and annihilation operators are known. In Ref. [19] the following expressions were found:

$$
\begin{aligned}
& \left\langle\hat{a}_{\omega, \mathbf{k}_{T}}^{R^{\dagger}} \hat{a}_{\omega^{\prime}, \mathbf{k}_{T}^{\prime}}^{R}\right\rangle=\frac{1}{\mathrm{e}^{\omega / T_{0}}-1} \delta\left(\omega-\omega^{\prime}\right) \delta^{2}\left(\mathbf{k}_{T}-\mathbf{k}_{T}^{\prime}\right), \\
& \left\langle\hat{a}_{\omega, \mathbf{k}_{T}}^{R} \hat{a}_{\omega^{\prime}, \mathbf{k}_{T}^{\prime}}^{R^{\dagger}}\right\rangle=\left(\frac{1}{\mathrm{e}^{\omega / T_{0}}-1}+1\right) \delta\left(\omega-\omega^{\prime}\right) \delta^{2}\left(\mathbf{k}_{T}-\mathbf{k}_{T}^{\prime}\right),
\end{aligned}
$$

$\left\langle\hat{a}_{\omega, \mathbf{k}_{T}}^{R} \hat{a}_{\omega^{\prime}, \mathbf{k}_{T}^{\prime}}^{R}\right\rangle=\left\langle\hat{a}_{\omega, \mathbf{k}_{T}}^{R \dagger} \hat{a}_{\omega^{\prime}, \mathbf{k}_{T}^{\prime}}^{R \dagger}\right\rangle=0$.

It was also shown that normal ordering with respect to the Rindler vacuum corresponds to neglecting the +1 in Eq. (24b), which arises from the commutation relations of creation and annihilation operators. From Eqs. (24a)-(24c), the following normally ordered expression can be calculated:

$$
\left\langle: \hat{\psi}^{2}:_{R}\right\rangle=\int_{0}^{+\infty} \mathrm{d} \omega \int_{\mathbb{R}^{2}} \mathrm{~d}^{2} k_{T}\left|u_{\omega, \mathbf{k}_{T}}\right|^{2} \frac{2}{\mathrm{e}^{\omega / T_{0}}-1},
$$

where the subscript $R$ stands for the normal ordering of Rindler creation and annihilation operators and corresponds to the subtraction of the expectation values in the Rindler vacuum. For a massless field, the above integration can be carried out analytically, yielding

$$
\left\langle: \hat{\psi}^{2}:_{R}\right\rangle=\frac{T_{0}^{2}}{12} \frac{1}{a^{2}\left(z^{\prime 2}-t^{2}\right)}=\frac{1}{12 \beta^{2}},
$$

where Eq. (15) has been used. Another useful expression which was obtained in Ref. [19] is

$$
\left\langle: u \cdot \partial \hat{\psi} u \cdot \partial \hat{\psi}:{ }_{R}\right\rangle=\frac{\pi^{2}}{30 \beta^{4}} .
$$




\section{ENTROPY CURRENT FOR A FREE SCALAR FIELD}

We are now in a position to calculate the thermodynamic potential current and the entropy current for a relativistic fluid at global thermodynamic equilibrium with acceleration in the RRW.

The basic ingredient to determine the entropy current, according to Eqs. (9) and (10), is the mean value of the stress-energy tensor. The general form of the mean value of a symmetric rank-two tensor at global thermodynamic equilibrium with acceleration is constrained by the symmetries of the density operator (19), as well as by the parameters at our disposal. In general, looking at Eq. (13), the mean value of any local operator $\hat{O}$ can be a function of $b, \varpi$, and $x$; however, the dependence on $x$ is constrained by the form of the density operator. Denoting by $\hat{\mathrm{T}}(x)$ the translation operator $\exp [i x \cdot \hat{P}]$, we have

$$
\begin{aligned}
\langle\hat{O}(x)\rangle & =\left\langle\hat{\mathrm{T}}(x) \hat{O}(0) \hat{\mathrm{T}}^{-1}(x)\right\rangle=\operatorname{tr}\left(\hat{\mathrm{T}}^{-1}(x) \hat{\rho} \hat{\mathrm{T}}(x) \hat{O}(0)\right) \\
& =\frac{1}{Z} \operatorname{tr}\left(\mathrm{e}^{-b \cdot \hat{P}+\frac{1}{2} \varpi: \hat{\top}^{-1}(x) \hat{\jmath} \hat{\mathrm{T}}(x)} \hat{O}(0)\right) \\
& =\frac{1}{Z} \operatorname{tr}\left(\mathrm{e}^{-\beta(x) \cdot \hat{P}+\frac{1}{2} \varpi: \hat{J}} \hat{O}(0)\right)=\langle\hat{O}(0)\rangle_{\beta(x)}
\end{aligned}
$$

where we have used the known relation from Poincaré algebra,

$$
\hat{\mathrm{T}}^{-1}(x) \hat{J}_{\mu \nu} \hat{\mathrm{T}}(x)=\hat{J}_{\mu \nu}-x_{\nu} \hat{P}_{\mu}+x_{\mu} \hat{P}_{\nu}
$$

and Eq. (12). Equation (26) implies that the expectation value of any local operator at global thermodynamic equilibrium depends on the space-time point only through the four-temperature vector field $\beta(x)$. Therefore, we can build up the most general expectation value of tensor fields of any rank just by combining $\beta$, the constant antisymmetric tensor $\varpi$, and the metric tensor $g$. Note that $\partial_{\mu} \beta_{\nu}=\varpi_{\nu \mu}$ and $\partial^{2} \beta=0$, so that derivatives of $\beta$ cannot enter as independent variables.

Likewise, any scalar function $F$, which is the mean value of a local operator or derived from the mean value of a local operator or derived from it, at global thermodynamic equilibrium with acceleration can only be a function of the two scalars formed with $\beta$ and $\varpi$, that is, $\beta^{2}=1 / T^{2}$ and $\alpha^{2}$, taking into account that $\alpha \cdot \beta=0$. Therefore a rank-two symmetric tensor such as the stress-energy tensor must have the following form:

$$
\left\langle\hat{T}^{\mu \nu}\right\rangle=F_{1} \beta^{\mu} \beta^{\nu}+F_{2} g^{\mu \nu}+F_{3} \alpha^{\mu} \alpha^{\nu}+F_{4}\left(\alpha^{\mu} \beta^{\nu}+\alpha^{\nu} \beta^{\mu}\right),
$$

with $F_{i}=F_{i}\left(\beta^{2}, \alpha^{2}\right)$. Furthermore, if the Hamiltonian $\hat{H}$ in Eq. (19) is invariant under time reversal, so is the density operator itself; as a consequence, the mixed components of the stress-energy tensor at $t=0$ must vanish, that is, $\left\langle\hat{T}^{0 i}(0, \mathbf{x})\right\rangle=0$. Since at $t=0$ we have $\alpha^{i} \neq 0$ and $\beta^{0} \neq 0$, the term proportional to $F_{4}$ in Eq. (27) breaks time-reversal invariance, and $F_{4}$ must then vanish. Similarly, it can be shown that the most general expression for the expectation value of a vector field should be of the simple form

$$
\left\langle\hat{V}^{\mu}\right\rangle=F\left(\beta^{2}, \alpha^{2}\right) \beta^{\mu},
$$

where the terms linear in $\alpha^{\mu}$ are forbidden by time-reversal invariance.

According to Eqs. (9) and (10), one also needs to determine the expectation value in the eigenvector of $\hat{\Upsilon}$ with the lowest eigenvalue. Looking at Eqs. (20) and (23), it is easy to realize that for a free field this eigenvector is just the Rindler vacuum $|0\rangle_{R}=\left|0_{R}\right\rangle \otimes\left|0_{L}\right\rangle$, whose eigenvalue is zero. Then, since it is not degenerate, the Rindler vacuum expectation value of any operator must have the same symmetries as its expectation value with the density operator (20), and we can thus write

$$
\begin{aligned}
\left\langle: \hat{T}^{\mu \nu}:_{R}\right\rangle & \equiv\left\langle\hat{T}^{\mu \nu}\right\rangle-{ }_{R}\left\langle 0\left|\hat{T}^{\mu \nu}\right| 0\right\rangle_{R} \\
& =F_{1} \beta^{\mu} \beta^{\nu}+F_{2} g^{\mu \nu}+F_{3} \alpha^{\mu} \alpha^{\nu} .
\end{aligned}
$$

Contracting Eq. (28) with the four-temperature twice, we obtain

$$
\begin{aligned}
\left\langle: \hat{T}^{\mu \nu}:_{R}\right\rangle \beta_{\nu} & =\left(F_{1} \beta^{2}+F_{2}\right) \beta^{\mu}, \\
F_{1} \beta^{2}+F_{2} & =\left\langle: \hat{T}^{\mu \nu}:_{R}\right\rangle \frac{\beta_{\mu} \beta_{\nu}}{\beta^{2}}=\left\langle: \hat{T}^{\mu \nu}:_{R}\right\rangle u_{\mu} u_{\nu}=\rho,
\end{aligned}
$$

where $\rho$ is the energy density. In summary, we have

$$
\left\langle: \hat{T}^{\mu \nu}:_{R}\right\rangle \beta_{\nu}=\rho \beta^{\mu}
$$

and, as a consequence,

$$
\phi^{\mu}=\beta^{\mu} \int_{1}^{+\infty} \mathrm{d} \lambda \rho(\lambda)
$$

with $\rho(\lambda)$ obtained from $\left\langle: \hat{T}^{\mu \nu}:_{R}\right\rangle(\lambda)$.

For the free real scalar field (22) we can obtain the canonical stress-energy tensor from the Lagrangian density,

$$
\mathcal{L}=\frac{1}{2} \partial_{\mu} \hat{\psi} \partial^{\mu} \hat{\psi}-\frac{1}{2} m^{2} \hat{\psi}^{2}
$$

that is,

$$
\hat{T}_{\mathrm{CAN}}^{\mu \nu}=\partial^{\mu} \hat{\psi} \partial^{\nu} \hat{\psi}-\frac{1}{4} \square \hat{\psi}^{2} g^{\mu \nu},
$$

where we have used the equations of motion $\left(\square+m^{2}\right) \hat{\psi}=0$. The energy density thus reads 
$\rho=\left\langle: \hat{T}^{\mu \nu}:_{R}\right\rangle u_{\mu} u_{\nu}=\left\langle: u \cdot \partial \hat{\psi} u \cdot \partial \hat{\psi}:_{R}\right\rangle-\frac{1}{4} \square\left\langle: \hat{\psi}^{2}:_{R}\right\rangle$.

By using the $\beta$ expression in Eq. (15), we obtain

$$
\square\left\langle: \hat{\psi}^{2}:_{R}\right\rangle=\frac{\alpha^{2}}{3 \beta^{4}},
$$

which, when plugged into Eq. (31) and combined with Eq. (25), gives the energy density of a free real scalar field in the RRW,

$$
\rho=\frac{\pi^{2}}{30 \beta^{4}}-\frac{\alpha^{2}}{12 \beta^{4}}
$$

This expression is precisely what was found in Refs. [21,26] with a perturbative expansion of the density operator (19) in $\alpha$ at order $\alpha^{2}$. Hence, we find that the perturbative series for the real scalar field is simply a polynomial in $\alpha$ of order 2 .

We now need the function $\rho(\lambda)$ to calculate the thermodynamic potential current. From Eqs. (6) and (19) it turns out that the introduction of the dimensionless parameter $\lambda$ corresponds to the rescaling $T_{0} \mapsto T_{0} / \lambda$. To make the full dependence of $\rho$ on $T_{0}$ apparent, it is convenient to introduce the Killing vector $\gamma \equiv T_{0} \beta$ which is independent of $T_{0}$ [see Eq. (15)] and, taking into account Eq. (18), write the above energy density as

$$
\rho=\frac{\pi^{2}}{30 \gamma^{4}} T_{0}^{4}+\frac{a^{2}}{12 \gamma^{4}} T_{0}^{2} .
$$

Rescaling $T_{0}$, we readily obtain

$$
\rho(\lambda)=\frac{\pi^{2}}{30 \gamma^{4}} \frac{T_{0}^{4}}{\lambda^{4}}+\frac{a^{2}}{12 \gamma^{4}} \frac{T_{0}^{2}}{\lambda^{2}}=\frac{\pi^{2}}{30 \beta^{4}} \frac{1}{\lambda^{4}}-\frac{\alpha^{2}}{12 \beta^{4}} \frac{1}{\lambda^{2}} .
$$

Plugging this into Eq. (29), we get the thermodynamic potential current

$$
\phi^{\mu}=\left(\frac{\pi^{2}}{90 \beta^{4}}-\frac{\alpha^{2}}{12 \beta^{4}}\right) \beta^{\mu}
$$

and, consequently, the entropy current in the RRW

$$
s^{\mu}=\left(\frac{2 \pi^{2}}{45 \beta^{4}}-\frac{\alpha^{2}}{6 \beta^{4}}\right) \beta^{\mu} .
$$

It should be pointed out that the above formulas depend on the stress-energy quantum operator. Indeed, for the improved stress-energy tensor, which is traceless for a massless field,

$$
\hat{T}_{\mathrm{IMP}}^{\mu \nu}=\hat{T}_{\mathrm{CAN}}^{\mu \nu}-\frac{1}{6}\left(\partial^{\mu} \partial^{\nu}-g^{\mu \nu} \square\right) \hat{\psi}^{2},
$$

we obtain a different expression for the energy density at equilibrium. This is an expected feature of thermodynamic equilibrium with rotation or acceleration, as was extensively discussed in Ref. [27]. Indeed, the additional term in the energy density pertaining to the canonical stress-energy tensor in Eq. (34) turns out to be

$$
\begin{aligned}
-\frac{1}{6}\left(u^{\mu} u^{\nu} \partial_{\mu} \partial_{\nu}-\square\right)\left\langle: \hat{\psi}^{2}:_{R}\right\rangle & =-\frac{1}{6}\left(u^{\mu} u^{\nu} \partial_{\mu} \partial_{\nu}-\square\right) \frac{1}{12 \beta^{2}} \\
& =\frac{\alpha^{2}}{12 \beta^{4}}
\end{aligned}
$$

as can be shown by using Eq. (15). Thus, adding the above contribution to Eq. (32), we find

$$
\rho_{\mathrm{IMP}}=\frac{\pi^{2}}{30 \beta^{4}},
$$

that is, the energy density calculated with the improved stress-energy tensor for the massless free real scalar field depends only on $\beta^{2}$ and not on $\alpha^{2}$, which is a somewhat surprising feature. Likewise, the entropy current gets modified and one is left with only the first term of Eq. (33),

$$
s_{\mathrm{IMP}}^{\mu}=\frac{2 \pi^{2}}{45 \beta^{4}} \beta^{\mu}
$$

\section{ENTANGLEMENT ENTROPY, AREA LAW, AND UNRUH EFFECT}

Equation (33) is the entropy current in the RRW, and therefore its integral on a space-like hypersurface whose boundary is the 2D surface $z^{\prime}=0, t=0$ (see Fig. 1) is

$$
S_{R}=\int_{z^{\prime}>0} \mathrm{~d} \Sigma_{\mu} s^{\mu}=-\operatorname{tr}_{R}\left(\hat{\rho}_{R} \log \hat{\rho}_{R}\right)
$$

according to Eqs. (20) and (21) and by means of the previous construction of the current. As the density operator is factorized, this entropy is also the entanglement entropy obtained by tracing out the field degrees of freedom in the LRW. At global thermodynamic equilibrium we have $\partial_{\mu} s^{\mu}=0$, and the entropy (36) can be calculated on any space-like hypersurface provided that the boundary flux vanishes. Indeed, this is the case for the RRW, as the timelike boundary is tangent to the entropy current (see Fig. 1).

A straightforward calculation of the entanglement entropy on the hypersurface $t=0$ with the canonical entropy current (33) yields

$$
S_{R}=\int_{\mathbb{R}^{2}} \mathrm{~d} x \mathrm{~d} y\left(\frac{2 \pi^{2}}{45}-\frac{\alpha^{2}}{6}\right) \frac{T_{0}^{3}}{a^{3}} \lim _{z^{\prime} \rightarrow 0} \frac{1}{2 z^{\prime 2}} .
$$




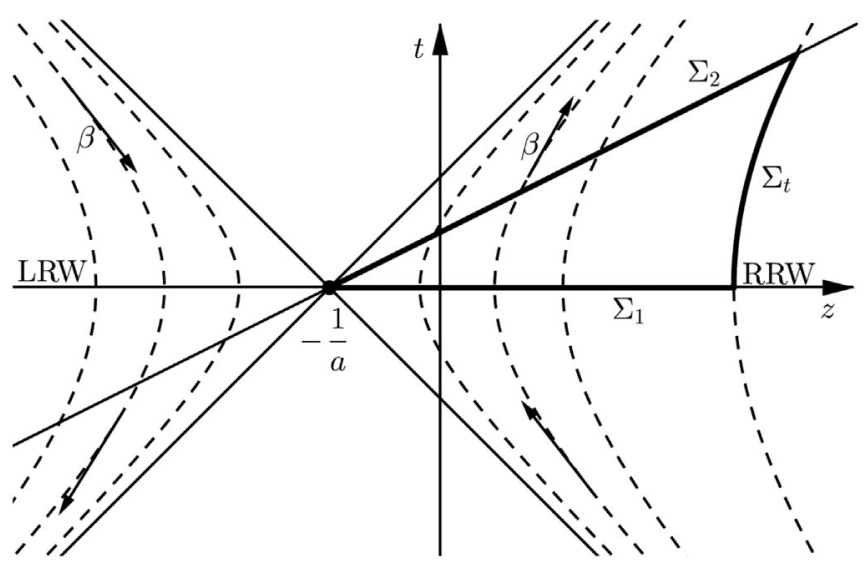

FIG. 1. Two-dimensional section of Minkowski space-time with the Killing field $\beta$ in Eq. (15) splitting the plane $t z$ into the Rindler wedges bounded by the light cone at $z=-1 / a$. The integrals of the conserved currents on the space-like hypersurfaces $\Sigma_{1}$ and $\Sigma_{2}$ in the RRW are the same due to Gauss' theorem and taking into account that the time-like hyperbolic boundary $\Sigma_{t}$ yields no contribution, as $\beta$ is perpendicular to its normal unit vector.

Thus, the entropy turns out to be proportional to the area of the $2 \mathrm{D}$ boundary surface separating the RRW from the LRW but with a divergent constant, owing to the fact that the comoving temperature $T$ diverges for $z^{\prime}=0$. This result is in full agreement with that of Bombelli et al. and Srednicki $[28,29]$.

The same result can be obtained with a more general and more elegant derivation which applies to general spacetimes. As the entropy current $s$ is divergenceless, $\nabla_{\mu} s^{\mu}=0$, it can be expressed as the Hodge dual of an exact threeform. If the domain is topologically contractible (as is the RRW), this form in turn can be expressed as the exterior derivative of a two-form [30-33], which eventually amounts to stating that the original vector field can be written as the divergence of an antisymmetric tensor field,

$$
s^{\mu}=\nabla_{\nu} \varsigma^{\mu \nu},
$$

whence, because of Stokes' theorem,

$$
\begin{aligned}
S & =\int_{\Sigma} \mathrm{d} \Sigma_{\mu} s^{\mu}=\int_{\Sigma} \mathrm{d} \Sigma_{\mu} \nabla_{\nu} \varsigma^{\mu \nu}=\frac{1}{2} \int_{\partial \Sigma} \mathrm{d} \tilde{S}_{\mu \nu} \varsigma^{\mu \nu} \\
& =-\frac{1}{4} \int_{\partial \Sigma} \mathrm{d} S^{\rho \sigma} \sqrt{-g} \epsilon_{\mu \nu \rho \sigma} \varsigma^{\mu \nu},
\end{aligned}
$$

where $\mathrm{d} S^{\rho \sigma}$ is the measure of the $2 \mathrm{D}$ boundary surface $\partial \Sigma$. Hence, the total entropy is expressed as a surface integral of a potential of a conserved current [30]. For our specific problem of equilibrium with acceleration, the general expression of the potential turns out to be

$$
\varsigma^{\mu \nu}=\frac{s}{2 \alpha^{2}}\left(\beta^{\mu} \alpha^{\nu}-\beta^{\nu} \alpha^{\mu}\right),
$$

where $s \equiv s^{\mu} u_{\mu}$ is the entropy density. Plugging Eq. (39) into Eq. (38), we get

$$
S=-\frac{1}{4} \int_{\partial \Sigma} \mathrm{d} S^{\rho \sigma} \sqrt{-g} \frac{s}{2 \alpha^{2}} \epsilon_{\mu \nu \rho \sigma}\left(\beta^{\mu} \alpha^{\nu}-\beta^{\nu} \alpha^{\mu}\right) .
$$

The boundary of the hypersurface $t=0$ is the plane $t=0$, $z^{\prime}=0$ and the plane $t=0, z^{\prime}=+\infty$. In the latter, the integrand vanishes for $s \propto z^{\prime-3}, \alpha^{2}$ is constant, and $\beta^{0} \alpha^{3} \propto z^{\prime}$. We are thus left with the $x y$ plane and, taking into account that the $\rho, \sigma$ indices can only take on the values 1,2 and the dependence of $\beta$ and $\alpha$ on $\left(z^{\prime}, t\right)$, we end up with Eq. (37).

A remarkable consequence of the entropy current method is the determination of the entanglement entropy in the Minkowski vacuum, when the state of the system is pure $\hat{\rho}=\left|0_{M}\right\rangle\left\langle 0_{M}\right|$. It is well known that [23]

$$
\hat{\rho}_{R}=\operatorname{tr}_{L}\left(\left|0_{M}\right\rangle\left\langle 0_{M}\right|\right)=\frac{1}{Z_{R}} \exp \left[-\frac{2 \pi}{a} \hat{\Pi}_{R}\right],
$$

that is, the Minkowski vacuum for a system with acceleration $a$ corresponds, in the RRW, to a mixed state with density operator (20) with $T_{0}=a / 2 \pi$, which is in essence the content of the Unruh effect. It was observed in Ref. [19] that, from a statistical thermodynamics viewpoint, this corresponds to a limiting comoving temperature of $T_{U}=|A| / 2 \pi$, where $|A|$ is the magnitude of the fouracceleration field. Because of Eq. (18), we thus have an upper bound for $\left|\alpha^{2}\right|=(2 \pi)^{2}$ in the Minkowski vacuum, and so Eq. (33) becomes

$$
s^{\mu}=\frac{32 \pi^{2}}{45} T_{U}^{3} u^{\mu},
$$

while for Eq. (35)

$$
s_{\mathrm{IMP}}^{\mu}=\frac{2 \pi^{2}}{45} T_{U}^{3} u^{\mu},
$$

which means that we have a nonvanishing entropy current in the Minkowski vacuum, owing to having traced out the field degrees of freedom in the LRW. Remarkably, the above expressions differ by a factor of 16, which is apparently an unexpected and odd feature. Yet, as it has been mentioned, at global thermodynamic equilibrium the mean value of the stress-energy tensor does depend on the specific quantum operator [in the case at hand, either Eq. (30) or Eq. (34)] and the entropy current as well. On the other hand, the total integrals like $\hat{P}^{\mu}$ and $\hat{J}^{\mu \nu}$ should not depend on it (see the discussion in Ref. [27]) and, as a consequence, the entanglement entropy should also be independent because $\hat{\rho}_{R}$ can be written as a trace over the field degrees of freedom of a density operator which is a function of the Poincaré generators [see Eq. (19)], 


$$
\hat{\rho}_{R}=\operatorname{tr}_{L}(\hat{\rho})=\frac{1}{Z} \operatorname{tr}_{L}\left(\exp \left[-\frac{\hat{H}}{T_{0}}+\frac{a}{T_{0}} \hat{K}_{z}\right]\right) .
$$

Nevertheless, the expressions for $\hat{\Pi}_{R}$ and $\hat{\Pi}_{L}$ [see Eqs. (20) and (21)] may inherit a dependence on the quantum stressenergy tensor because of the truncation at $z^{\prime}=0$. This issue will be the subject of further investigation.

\section{SUMMARY AND OUTLOOK}

In summary, we have presented the condition of existence of an entropy current and a general method to calculate it. An entropy current can be obtained if the spectrum of the local equilibrium operator-which boils down to the Hamiltonian multiplied by $1 / T$ in the simplest case of global homogeneous equilibrium - is bounded from below. We have applied our method to the case of a fluid with a comoving acceleration of constant magnitude, which is a known instance of nontrivial global thermodynamic equilibrium in Minkowski space-time. We have also shown its connection to the entanglement entropy in the vacuum, and its relation with the Unruh effect. Furthermore, we have shown that, at least at global equilibrium, the total entropy can be expressed as a surface integral, in agreement with Ref. [30]. We expect this method to be applicable to other problems where the total entropy has to be determined, like, e.g., relativistic hydrodynamics or thermodynamic equilibrium in general curved space-time.

The entropy current is expectedly dependent on the specific form of the stress-energy tensor operator. Besides, for the free scalar field, it seems that the total entanglement entropy also depends on the particular stress-energy tensor operator. This is a subject for future studies.

\section{ACKNOWLEDGMENTS}

We acknowledge useful discussions with D. Seminara.

\section{APPENDIX: CALCULATION OF THE ENTROPY POTENTIAL}

The search for a potential for the entropy current uses the same method as for the stress-energy tensor. To form an antisymmetric tensor we can just use the four-vectors $\beta$ and $\alpha$, and hence the only possible combination is

$$
\alpha^{\mu} \beta^{\nu}-\alpha^{\nu} \beta^{\mu}
$$

In turn, this is just proportional to the thermal vorticity, according to Eq. (17), so we can write the general form of the potential as

$$
\varsigma^{\mu \nu}=G\left(\beta^{2}, \alpha^{2}\right) \varpi^{\mu \nu},
$$

where $G$ is a general scalar function such that

$$
s^{\mu}=\partial_{\nu}\left(G \varpi^{\mu \nu}\right) .
$$

By introducing the proper entropy density $s$ such that $s^{\mu} u_{\mu}=s$, we have

$$
s=s^{\mu} u_{\mu}=\frac{1}{\sqrt{\beta^{2}}} s^{\mu} \beta_{\mu}=\frac{1}{\sqrt{\beta^{2}}} \beta_{\mu} \varpi^{\mu \nu} \partial_{\nu} G,
$$

as $\varpi$ is constant. Now, $\varpi^{\mu \nu} \beta_{\mu}=-\sqrt{\beta^{2}} \alpha^{\nu}$ from Eq. (17), and so

$$
s=-\alpha^{\nu} \partial_{\nu} G=-\alpha^{\nu} \partial_{\nu} \beta^{2} \frac{\partial G}{\partial \beta^{2}}
$$

because $\alpha^{2}$ is a constant in the pure acceleration case. The Killing equation (11) implies [21]

$$
\partial_{\nu} \beta^{2}=-2 \sqrt{\beta^{2}} \alpha_{\nu}
$$

so that Eq. (A2) becomes

$$
s=2 \alpha^{2} \sqrt{\beta^{2}} \frac{\partial G}{\partial \beta^{2}},
$$

whose solution is

$$
G=\int \mathrm{d} \beta^{2} \frac{s}{2 \alpha^{2}} \frac{1}{\sqrt{\beta^{2}}} .
$$

For the massless case, we have $s=C(\alpha)^{2} /{\sqrt{\beta^{2}}}^{3}$ from Eq. (33), and thus

$$
G=\frac{C\left(\alpha^{2}\right)}{2 \alpha^{2}} \int \mathrm{d} \beta^{2} \frac{1}{\beta^{4}}=-\frac{C\left(\alpha^{2}\right)}{2 \alpha^{2}} \frac{1}{\beta^{2}}=-s \frac{2 \alpha^{2}}{\sqrt{\beta^{2}}} .
$$

Plugging the above result into Eq. (A1) and using Eq. (17), we obtain

$$
\begin{aligned}
\varsigma^{\mu \nu} & =-\frac{s}{2 \alpha^{2}} \sqrt{\beta^{2}} \varpi^{\mu \nu}=-\frac{s}{2 \alpha^{2}} \sqrt{\beta^{2}}\left(\alpha^{\mu} u^{\nu}-\alpha^{\nu} u^{\mu}\right) \\
& =\frac{s}{2 \alpha^{2}}\left(\alpha^{\nu} \beta^{\mu}-\alpha^{\mu} \beta^{\nu}\right),
\end{aligned}
$$

which is precisely Eq. (39). 
[1] W. Israel, Ann. Phys. (N.Y.) 100, 310 (1976).

[2] R. Loganayagam, J. High Energy Phys. 05 (2008) 087.

[3] J. Bhattacharya, S. Bhattacharyya, and M. Rangamani, J. High Energy Phys. 02 (2013) 153.

[4] C. Chattopadhyay, A. Jaiswal, S. Pal, and R. Ryblewski, Phys. Rev. C 91, 024917 (2015).

[5] R. Banerjee, S. Dey, and B. R. Majhi, Phys. Rev. D 92 , 044019 (2015).

[6] P. Glorioso, M. Crossley, and H. Liu, J. High Energy Phys. 09 (2017) 096.

[7] F. M. Haehl, R. Loganayagam, and M. Rangamani, J. High Energy Phys. 04 (2016) 039.

[8] K. Jensen, R. Marjieh, N. Pinzani-Fokeeva, and A. Yarom, J. High Energy Phys. 01 (2019) 061.

[9] K. Hattori, M. Hongo, X. G. Huang, M. Matsuo, and H. Taya, Phys. Lett. B 795, 100 (2019).

[10] K. Jensen, M. Kaminski, P. Kovtun, R. Meyer, A. Ritz, and A. Yarom, Phys. Rev. Lett. 109, 101601 (2012).

[11] S. Bhattacharyya, J. High Energy Phys. 08 (2014) 165.

[12] S. Bhattacharyya, J. High Energy Phys. 07 (2014) 139.

[13] F. M. Haehl, R. Loganayagam, and M. Rangamani, Phys. Rev. Lett. 121, 051602 (2018).

[14] D. N. Zubarev, A. V. Prozorkevich, and S. A. Smolyanskii, Theor. Math. Phys. 40, 821 (1979).

[15] Ch. G. Van Weert, Ann. Phys. (N.Y.) 140, 133 (1982).

[16] F. Becattini, L. Bucciantini, E. Grossi, and L. Tinti, Eur. Phys. J. C 75, 191 (2015).
[17] T. Hayata, Y. Hidaka, T. Noumi, and M. Hongo, Phys. Rev. D 92, 065008 (2015).

[18] M. Buzzegoli and F. Becattini, J. High Energy Phys. 12 (2018) 002.

[19] F. Becattini, Phys. Rev. D 97, 085013 (2018).

[20] F. Becattini, Phys. Rev. Lett. 108, 244502 (2012).

[21] M. Buzzegoli, E. Grossi, and F. Becattini, J. High Energy Phys. 10 (2017) 091; 07 (2018) 119.

[22] J. I. Korsbakken and J. M. Leinaas, Phys. Rev. D 70, 084016 (2004).

[23] L. C. B. Crispino, A. Higuchi, and G. E. A. Matsas, Rev. Mod. Phys. 80, 787 (2008).

[24] S. A. Fulling, Phys. Rev. D 7, 2850 (1973).

[25] W. G. Unruh, Phys. Rev. D 14, 870 (1976).

[26] F. Becattini and E. Grossi, Phys. Rev. D 92, 045037 (2015).

[27] F. Becattini and L. Tinti, Phys. Rev. D 84, 025013 (2011).

[28] L. Bombelli, R. K. Koul, J. Lee, and R. D. Sorkin, Phys. Rev. D 34, 373 (1986).

[29] M. Srednicki, Phys. Rev. Lett. 71, 666 (1993).

[30] R. M. Wald, Phys. Rev. D 48, R3427 (1993).

[31] C. Eling, A. Meyer, and Y. Oz, J. High Energy Phys. 08 (2012) 088.

[32] B. R. Majhi and T. Padmanabhan, Phys. Rev. D 85, 084040 (2012).

[33] B. R. Majhi and T. Padmanabhan, Phys. Rev. D 86, 101501 (2012). 